Mosteiro García, M.J. y Porto Castro, A.M. (2017). Análisis de los estereotipos de género en alumnado de formación profesional: diferencias según sex, edad y grado. Revista de Investigación Educativa, 35(1), 151-165 DOI: http://dx.doi.org/10.6018/rie.35.1.257191

\title{
Análisis de los Estereotipos de Género en alumnado de Formación Profesional: Diferencias según sexo, edad y grado
}

\section{Analyses of Gender Stereotypes in Vocational Education students: Differences according to sex, age and grade}

\author{
María Josefa Mosteiro García y Ana María Porto Castro \\ Universidad de Santiago de Compostela, Departamento de Métodos de Investigación y Diagnóstico en Educación, \\ Facultad de Ciencias de la Educación
}

\begin{abstract}
Resumen
Los estereotipos de género, creencias culturales acerca de los rasgos que poseen hombres y mujeres influyen en la forma de actuar de las personas y sus relaciones con los demás. Este trabajo tiene como objetivo conocer la presencia de estereotipos de género en alumnado de Formación Profesional y comprobar si existen diferencias en función del sexo, la edad y el grado que cursan. Para dar respuesta a estos objetivos se realizó un estudio descriptivo, utilizando una escala para recoger la opinión de una muestra de 452 estudiantes que cursan primer año de ciclos formativos de Grado Medio y Superior de la Comunidad Autónoma de Galicia. Los resultados obtenidos, tras la aplicación de la prueba $t$ y el análisis de varianza, muestran un rechazo de las y los jóvenes hacia los estereotipos de género, encontrando diferencias por sexo y ciclo formativo. De estos resultados se deduce que, a pesar de los cambios que se han producido en los últimos años, es necesario seguir sensibilizando al alumnado en materia de igualdad para poder lograr una sociedad más justa e igualitaria.

Palabras clave: género; estereotipos de género; formación profesional; educación en igualdad

Correspondencia: Ana María Porto Castro, anamaria.porto@usc.es, Universidad de Santiago de Compostela, Departamento de Métodos de Investigación y Diagnóstico en Educación, Facultad de Ciencias de la Educación, Rúa Xosé María Suárez Núñez, s/n (Campus Vida), 15782, Santiago de Compostela.
\end{abstract}




\begin{abstract}
Gender stereotypes, defined as beliefs on the different characteristics of women and men in our society, play a key role in the ways of thinking, interpreting and acting subjects, and how to relate to other people. In this paper we are interested in knowing the presence of gender stereotypes in vocational education students and see if there are differences according to sex, age and training course enrollment. To meet these objectives a descriptive study was made with a sample of 452 students attending first year of Intermediate and Higher training cycles in Galicia. The results, after application of the t-test and analysis of variance show a rejection of young people and adolescents to gender stereotypes, finding differences by sex and training cycle. From these results it follows that even though something is changing in society, it is necessary to further sensitize the students equal to move towards a more just and egalitarian society.

Keywords: gender; gender stereotypes; vocational education; education for equality
\end{abstract}

\title{
Introducción
}

El estudio de las diferencias entre los sexos, cuestión de interés universal, se inicia a principios del siglo $X X$, si bien, las primeras investigaciones se realizan a mediados del XIX, a raíz de los cambios socio-económicos de la Revolución Industrial y a los enormes progresos en el campo científico en general.

Estos estudios han ido evolucionando desde sus inicios y han centrado su interés en distintos ámbitos, desde las diferencias estrictamente psicológicas entre hombres y mujeres, hasta los patrones de conducta característicamente masculinos y femeninos. En todos ellos subyace la idea de que el género es un organizador básico en cualquier cultura y sociedad.

En función de las características sexuales externas y de las diferentes funciones dentro del proceso reproductivo, hombres y mujeres ocupan roles diferenciados en el hacer social. Esta diferenciación ha sido interpretada de forma jerárquica en nuestra tradición cultural, entendiendo que las mujeres son inferiores a los hombres, y originando, de este modo, modelos de desigualdad entre ambos sexos.

Además, esta visión del género ha dado lugar a una diferenciación en la socialización de hombres y mujeres. Tal y como indican Colás y Villaciervos (2007, p. 38):

Las personas se convierten en hombres y mujeres en función del aprendizaje de representaciones culturales de género que rigen, no sólo, su constitución genérica, sino también el carácter de las relaciones que, unos y otras, mantienen en diferentes esferas sociales (en ámbitos como la familia, la escuela, el grupo de iguales, etc.). Así, el género como sistema cultural, provee de referentes culturales que son reconocidos y asumidos por las personas.

Mediante los procesos de socialización, los individuos van conformando una identidad de género determinada y van aprendiendo las conductas, las normas, las costumbres, los estereotipos y los roles a partir de los patrones que dicta la cultura, según sean varones o mujeres. 
En los últimos años se ha producido un cambio en los roles de género. Uno de los hechos que ha contribuido de manera singular a este cambio ha sido el paulatino y creciente nivel educativo alcanzado por las mujeres que poco a poco se ha ido equiparando, e incluso en algunos casos, ha superado al logrado por los varones.

Es obvio que el incremento de la formación académica de las mujeres ha favorecido su incorporación al mercado de trabajo y sus posibilidades de promoción y participación en la vida económica y social.

Ahora bien, este hecho que ha supuesto un cambio importante en la vida de las mujeres no lleva consigo, necesariamente, la desaparición de los estereotipos de género. Hoy en día todavía siguen vigentes creencias estereotipadas acerca del trabajo de mujeres y hombres, consecuencia de los diferentes roles que uno y otro sexo desempeñan en la sociedad.

El presente trabajo pretende conocer si en las y los jóvenes en formación persisten ciertos estereotipos de género, y comprobar si la interiorización de los mismos difiere en función del sexo, la edad y el grado de Formación Profesional cursado.

\section{Los estereotipos de género}

Para aproximarnos al concepto de estereotipos de género conviene, previamente, hacer referencia a lo que se entiende por sexo y género. El sexo alude a las diferencias estrictamente biológicas que existen entre mujeres y hombres mientras que el género se refiere a las características consideradas socialmente apropiadas para uno y otro sexo en cada sociedad determinada.

El género es, por tanto, una "representación cultural, que contiene ideas, prejuicios, valores, interpretaciones, normas, deberes, mandatos y prohibiciones sobre la vida de las mujeres y de los hombres (Colás \& Villaciervos, 2007, p. 37).

Los estereotipos de género son creencias socialmente aceptadas acerca de los rasgos y roles característicos de uno y otro sexo en la sociedad (de Lemus, Castillo, Moya, Padilla, \& Ryan, 2008).

Estas creencias llevan implícita la existencia de una dicotomía de rasgos de personalidad, roles, características físicas y ocupaciones que se asocian tradicionalmente a hombres y mujeres y tienen una gran influencia en el individuo, en su percepción del mundo y de sí mismo y en su conducta (Porto et al., 2012, p. 3).

Esto es, la interiorización de las diferencias de género influye en la forma de actuar de las personas y sus relaciones con los demás.

Los estereotipos de género son fiel reflejo de una cultura y una historia y, como tal, tienden a mantenerse porque responden a las necesidades de la sociedad de preservar las normas sociales que mantienen el statu quo. En este sentido, se caracterizan por su fuerte resistencia al cambio y por seguir vigentes en la sociedad hasta que se produzca un cambio en los roles de mujeres y hombres.

Estas creencias estereotipadas se transmiten de una generación a otra a través de un complejo sistema de interacciones en el que intervienen diversos agentes e institu- 
ciones. Los agentes de socialización juegan un papel fundamental en la transmisión y perpetuación de los estereotipos de género tradicionales (Vázquez-Cupeiro, 2015). La familia y la escuela son los principales espacios de socialización en los que se aprenden e interiorizan los roles masculinos y femeninos (Perry \& Pauletti, 2011).

La manera de actuar de los/as progenitores con sus hijos/as incide en la adquisición y consolidación de los estereotipos de género. El diferente trato que se da en la familia a niños y niñas sirve como modelo en la interiorización de los roles que se consideran propios de uno y otro sexo.

Según las teorías de la perspectiva cognitiva, desarrolladas dentro del marco general de las teorías de los esquemas (Bem, 1981; Markus, Crane, Bernstein \& Siladi, 1982), el concepto de yo de género influye en el procesamiento de la información relacionada con las categorías sexuales. De este modo, las niñas y los niños empiezan a adquirir los estereotipos de género casi al mismo tiempo que toman conciencia de su identidad de género.

Las pautas diferenciales de socialización que tienen lugar desde edades muy tempranas en la vida del individuo, van a tener implicaciones en la etapa adulta y dan respuesta a las diferencias en las conductas, motivaciones, valores y autoncepto entre uno y otro sexo.

Por otro lado, la escuela a través del currículum oculto, refuerza los estereotipos de género que se inician en el seno familiar, estereotipos que tienen una gran influencia en el autoconcepto y logro académico (Igbo, Onu, \& Obiyo, 2015).

De acuerdo con Elejabeitia y López Sáez (2003, p. 182) es importante señalar que

los estereotipos son la base de los prejuicios que afectan tanto a hombres como a mujeres por el carácter que tienen no solo descriptivo sino prescriptivo. Cada vez que la persona aprende que es 'niño' o que es 'niña', va aprendiendo también cual es la conducta apropiada para esa autocategorización, siguiendo las prescripciones culturales asociadas a su sexo.

Cuando los hombres o las mujeres no cumplen con lo socialmente esperable para su sexo, sufren las consecuencias que impone la sociedad y sus actuaciones suelen ser criticadas y rechazadas socialmente.

En los últimos años, diversos estudios (Alvariñas, Fernández Villarino, \& López Villar, 2009; Alvariñas \& Novoa, 2016; Castillo-Mayén \& Montes-Berges, 2014; Colás \& Villaciervos, 2007; Delpino \& Eresta, 2013; Díaz-Aguado, Martínez Arias, \& Martínez Babarro, 2014; Heilman, 2015; Kurtz-Costes, Copping, Rowley, \& Kinlaw, 2014; Miller, Eagly, \& Linn, 2015; Pastor \& Marrrucci, 2015; Rodríguez San Julián \& Megías, 2015; Ruiz Palomino, Ballester, Gil Larrio, Giménez García, \& Salmerón 2010; Sánchez García et al. 2011) han tratado de conocer la persistencia de estereotipos de género en alumnado de distintas etapas educativas.

Así, Miller et al. (2015) constatan que chicos y chicas siguen teniendo una imagen estereotipada de las profesiones y mantienen la creencia de que los hombres tienen cualidades más apropiadas para desempeñar profesiones en el ámbito de la ciencia, siendo la estereotipia más acusada en el caso del sexo masculino. Destacar que, precisamente el carácter prescriptivo de los estereotipos de género impide el desarrollo profesional de las mujeres (Heilman, 2015). 
En la misma línea, según Kurtz-Costes et al. (2014) las creencias estereotipadas de los adultos sobre las diferentes capacidades académicas de niños y niñas condicionan sus elecciones futuras.

Por su parte Colás y Villaciervos (2007) en su estudio realizado con alumnado de secundaria verifican una elevada interiorización de conductas estereotipadas hacia el otro sexo entre el alumnado. Asimismo, Alvariñas et al. (2009) evidencian la existencia de estereotipos en el deporte, aunque también muestran cierta progresión respecto a algunas cuestiones, lo que hace pensar que el pensamiento de las y los jóvenes está evolucionando positivamente.

Sánchez García et al. (2011) señalan que a mayor edad y nivel educativo menor presencia de estereotipos de género en las personas y viceversa, a menor edad y nivel educativo mayor presencia de estereotipia de género. La flexibilidad de los estereotipos de género con la edad también se pone de manifiesto en el estudio realizado por Ruiz Palomino et al. (2010), quienes comprobaron que los y las adolescentes manifiestan actitudes discriminatorias en función del género, creencias que van cambiando a medida que pasa el tiempo.

García Pérez et al. (2010) en una muestra de estudiantes de primaria y secundaria de centros públicos y privados de la provincia de Sevilla, comprobaron que las alumnas están más sensibilizadas en materia de igualdad que los alumnos. También constataron que el alumnado muestra conductas más igualitarias hacia cuestiones relacionadas con actividades profesionales y sociales que personales, sobre todo en lo que se refiere a la reproducción y a las tareas de cuidado, actividades tradicionalmente asociadas al rol femenino.

En un trabajo realizado con estudiantes de educación secundaria de Italia y España Pastor y Marrucci (2015) constataron que, en general, el alumnado español está más sensibilizado en materia de igualdad que el italiano. Por otra parte, observaron que las alumnas españolas están más sensibilizadas en materia de igualdad que los alumnos, especialmente en las conductas que tienen que ver con el plano social y personal, puesto que en estos ámbitos es donde existe una mayor desigualdad entre uno y otro sexo.

El aumento del rechazo del sexismo es constatado por Díaz-Aguado et al. (2015) en estudiantes de Educación Secundaria Obligatoria, Bachillerato y Ciclos Formativos de centros de distintas Comunidades Autónomas.

Según Delpino y Eresta (2013), todavía persisten en los adolescentes ciertos estereotipos tradicionales en el terreno de la sexualidad que inciden probablemente en las formas en las que los/as adolescentes encaran las relaciones de pareja, que en ocasiones incluyen ingredientes de violencia.

Para Rodríguez San Julián y Megías (2015), sigue existiendo una clara diferenciación en las atribuciones que chicos y chicas conceden a uno y otro sexo. Así constatan en su trabajo que los rasgos de sensibilidad, comprensión, inteligencia, responsabilidad y prudencia son asociados al sexo femenino, mientras lo masculino se relaciona con el dinamismo y la funcionalidad, la actuación y el protagonismo.

En la misma línea, pero en el ámbito universitario, las conclusiones obtenidas por Castillo-Mayén y Montes-Berges (2014) subrayan que, si bien se observan importantes cambios en los estereotipos de género, especialmente en los estereotipos femeninos, todavía se mantienen ciertas conductas estereotipadas que perpetúan las desigualdades entre uno y otro sexo. 
Por su parte, Alvariñas y Novoa (2016) confirman la resistencia al cambio del estereotipo que afirma que unos deportes son más apropiados para hombres y otros para mujeres, aunque sugieren un cierto avance en relación con los estereotipos tradicionales.

A la vista de los resultados de estos estudios, todo parece indicar que siguen vigentes los estereotipos de género entre las y los jóvenes y adolescentes. Debido al papel que éstos juegan en las formas de pensar, de interpretar y de actuar de las personas y en su relación con los demás así como su importancia educativa y personal, decidimos realizar el presente estudio y presentar, a continuación, los resultados obtenidos al analizar el grado de acuerdo o desacuerdo de una muestra de estudiantes de Formación Profesional, ante una serie de afirmaciones estereotipadas.

\section{Método}

\section{Objetivo}

Nuestro objetivo es conocer la presencia de estereotipos de género en el alumnado que cursa ciclos formativos de Grado Medio y Superior en la Comunidad Autónoma de Galicia y comprobar si existen diferencias en función del sexo, la edad y si el ciclo cursado es de grado medio o superior.

\section{Población y Muestra}

La población está constituida por alumnado de Formación Profesional de Grado Medio y Superior que cursa sus estudios en centros educativos públicos, concertados y privados de la Comunidad Autónoma Gallega.

La muestra, seleccionada al azar, está formada por un total de 452 estudiantes, 215 hombres $(47.6 \%)$ y 237 mujeres $(52.4 \%)$ de edades comprendidas mayoritariamente entre los 16 y 20 años (62.3\%), que estudian primer (92.9\%) y segundo curso $(7.1 \%)$ de ciclos formativos de grado medio (53.3\%) y superior (46.7\%) en Institutos de Educación Secundaria de las provincias de Pontevedra (50.4\%) y A Coruña (49.6\%).

\section{Instrumento}

Se diseñó una escala ad hoc tipo Likert -ver tabla 1- compuesta por un total de 13 ítems, con cinco opciones de respuesta (1: Muy en desacuerdo a 5: Muy de acuerdo) para medir el grado de acuerdo del alumnado ante un conjunto de afirmaciones estereotipadas. En el instrumento aparecen también recogidas las variables de identificación personal sexo (hombre, mujer), ciclo formativo (ciclo formativo grado medio y superior), edad (16-20 años, 21-25 años, 26-30 años, 31-35 años, 36-45 años y más de 45 años) y curso $\left(1^{\circ}\right.$ y $\left.2^{\circ}\right)$.

La escala se sometió al análisis y valoración de un grupo de expertos/as en género del ámbito universitario. Como resultado de las contribuciones otorgadas por los/as expertos/as se reformuló la redacción de varios de los ítems, quedando la escala definitiva configurada por el mismo número de ítems de la versión inicial. 
El cálculo de la fiabilidad del instrumento de medida a través del coeficiente Alpha de Cronbach proporciona un valor de .830 informando que posee una elevada consistencia interna.

Tabla 1

Escala para evaluar los estereotipos de Género

Indica en qué medida estás de acuerdo con las siguientes afirmaciones (1) Muy en desacuerdo; (2) En desacuerdo; (3) Indiferente; (4) De acuerdo; (5) Muy de acuerdo.

1. Las mujeres deben tener las mismas posibilidades de trabajo que los hombres

2. Contratar a las mujeres solo trae problemas a las empresas

3. Hay trabajos que son más apropiados para las mujeres que para los hombres

4. Hay trabajos que son más apropiados para los hombres que para las mujeres

5. Hay trabajos en los que los hombres deberían tener preferencia sobre las mujeres a la hora de los ascensos y de la promoción

6. Las mujeres tienen más dificultades para encontrar un trabajo que tradicionalmente se identifica como un trabajo de hombres

7. Los hombres tienen más dificultades para encontrar un trabajo que tradicionalmente se identifica como un trabajo de mujeres

8. Las ocupaciones en las que trabajan sobre todo hombres tienen mayor reconocimiento social que las ocupaciones en las que la presencia femenina es mayoritaria

9. Las ocupaciones en las que trabajan sobre todo mujeres poseen peores condiciones laborales que las ocupaciones en las que trabajan sobre todo hombres

10. Las profesiones en las que trabajan sobre todo mujeres están peor remuneradas que las profesiones en las que trabajan sobre todo hombres

11. Hay trabajos en los que se necesita fuerza y otras cualidades/habilidades que las mujeres no tienen

12. Hay algunos tipos de trabajos más apropiados para las mujeres y otros más apropiados para los hombres

13. En nuestra sociedad las mujeres no sufren discriminación a la hora de incorporarse al mercado laboral

\section{Procedimiento de recogida y análisis de datos}

El instrumento fue aplicado por los miembros del equipo de investigación, que recibieron instrucciones generales a seguir en el momento de la administración de la escala, con el fin de que las condiciones de aplicación fuesen lo más homogéneas posibles, tratando de este modo de asegurar la validez del procedimiento.

Previa autorización, la escala fue aplicada, durante el mismo periodo de tiempo, en el aula y en el horario académico habitual. Se informó brevemente a los sujetos que 
voluntariamente decidieron participar en la investigación, de los objetivos del estudio, al mismo tiempo que se le dieron las instrucciones específicas necesarias para responder a las cuestiones planteadas.

El tratamiento de los datos se realizó mediante el paquete estadístico SPSS. Se llevó a cabo un análisis descriptivo básico (media, desviación típica), se aplicó la prueba $t$ de Student para comprobar la existencia de diferencias estadísticamente significativas en los estereotipos de género en función del sexo y si el ciclo formativo cursado es de grado medio o superior y el análisis de la varianza para conocer la existencia de diferencias en función de la edad.

\section{Resultados}

A continuación, presentamos los resultados obtenidos aludiendo en primer lugar, a los estereotipos de género de la muestra total para, a continuación, mostrar los alcanzados en función del sexo, la edad y ciclo formativo de grado medio y superior.

\section{Estereotipos de género en la muestra total}

Tal y como se puede comprobar en la tabla 2, el alumnado participante en el estudio manifiesta muy claramente su grado de acuerdo ante la afirmación "Las mujeres deben tener las mismas posibilidades de trabajo que los hombres". Por otra parte, también se muestra de acuerdo, aunque en menor medida, ante las afirmaciones "Las mujeres tienen más dificultades para encontrar un trabajo que tradicionalmente se identifica como un trabajo de hombres", "Los hombres tienen más dificultades para encontrar un trabajo que tradicionalmente se identifica como un trabajo de mujeres" y "Las profesiones en las que trabajan sobre todo mujeres están peor remuneradas que las profesiones en las que trabajan sobre todo hombres".

Tabla 2

Estereotipos de género en la muestra total

\begin{tabular}{lcc}
\hline Estereotipos de Género & $(\bar{X})$ & $(S)$ \\
\hline Las mujeres deben tener las mismas posibilidades de trabajo que los hombres. & 4.66 & 1.022 \\
Contratar a las mujeres solo trae problemas a las empresas. & 1.27 & 0.852 \\
Hay trabajos que son más apropiados para las mujeres que para los hombres. & 2.53 & 1.367 \\
$\begin{array}{l}\text { Hay trabajos que son más apropiados para los hombres que para las mujeres. } \\
\text { Hay trabajos en los que los hombres deberían tener preferencia sobre las mu- }\end{array}$ & 1.46 & 1.436 \\
$\begin{array}{l}\text { jeres a la hora de los ascensos y de la promoción. } \\
\begin{array}{l}\text { Las mujeres tienen más dificultades para encontrar un trabajo que tradiciona- } \\
\text { lmente se identifica como un trabajo de hombres. }\end{array}\end{array}$ & 3.86 & 1.261 \\
\hline
\end{tabular}




\begin{tabular}{lcc}
\hline $\begin{array}{l}\text { Los hombres tienen más dificultades para encontrar un trabajo que tradiciona- } \\
\text { lmente se identifica como un trabajo de mujeres. }\end{array}$ & 3.31 & 1.348 \\
$\begin{array}{l}\text { Las ocupaciones en las que trabajan sobre todo hombres tienen mayor recono- } \\
\text { cimiento social que las ocupaciones en las que la presencia femenina es may- } \\
\text { oritaria. }\end{array}$ & 2.94 & 1.360 \\
$\begin{array}{l}\text { Las ocupaciones en las que trabajan sobre todo mujeres poseen peores condi- } \\
\text { ciones laborales que las ocupaciones en las que trabajan sobre todo hombres. }\end{array}$ & 2.74 & 1.364 \\
$\begin{array}{l}\text { Las profesiones en las que trabajan sobre todo mujeres están peor remunera- } \\
\text { das que las profesiones en las que trabajan sobre todo hombres. }\end{array}$ & 3.17 & 1.354 \\
$\begin{array}{l}\text { Hay trabajos en los que se necesita fuerza y otras cualidades/habilidades que } \\
\text { las mujeres no tienen. }\end{array}$ & 2.97 & 1.435 \\
$\begin{array}{l}\text { Hay algunos tipos de trabajos más apropiados para las mujeres y otros más } \\
\text { apropiados para los hombres. }\end{array}$ & 3.08 & 1.447 \\
$\begin{array}{l}\text { En nuestra sociedad las mujeres no sufren discriminación a la hora de incor- } \\
\text { porarse al mercado laboral. }\end{array}$ & 2.95 & 1.400 \\
\hline
\end{tabular}

El alumnado encuestado muestra la persistencia de determinados estereotipos y no se posiciona a favor ni en contra de afirmaciones como las siguientes: "Hay algunos tipos de trabajos más apropiados para las mujeres y otros más apropiados para los hombres", "En nuestra sociedad las mujeres no sufren discriminación a la hora de incorporarse al mercado laboral", "Hay trabajos en los que se necesita fuerza y otras cualidades/habilidades que las mujeres no tienen" y "Las ocupaciones en las que trabajan sobre todo hombres tienen mayor reconocimiento social que las ocupaciones en las que la presencia femenina es mayoritaria".

Las respuestas del alumnado a las afirmaciones "Contratar a las mujeres sólo trae problemas a las empresas" y "Hay trabajos en los que los hombres deberían tener preferencia sobre las mujeres a la hora de los ascensos y de la promoción" alcanzan las medias más bajas, manifestando las y los participantes en el estudio menor grado de acuerdo ante las mismas.

\section{Diferencias por sexo en los estereotipos de género}

$\mathrm{Al}$ analizar los datos obtenidos en función del sexo, los resultados derivados de la prueba $t$ de Student muestran la existencia de diferencias estadísticamente significativas en todos los ítems de la escala entre uno y otro sexo.

Así, las chicas alcanzan puntuaciones medias superiores a las de los chicos, con valores muy próximos al máximo de la escala, ante afirmaciones como las siguientes: “Las mujeres deben tener las mismas posibilidades de trabajo que los hombres" y "Las mujeres tienen más dificultades para encontrar un trabajo que tradicionalmente se identifica como un trabajo de hombres", lo que significa que están más de acuerdo que sus compañeros en dichas afirmaciones.

Se sitúan también con puntuaciones medias superiores a las de sus compañeros, aunque en menor medida, ante las afirmaciones: “Los hombres tienen más dificultades para encontrar un trabajo que tradicionalmente se identifica como un trabajo de muje- 
res", "Las ocupaciones en las que trabajan sobre todo hombres tienen mayor reconocimiento social que las ocupaciones en las que la presencia femenina es mayoritaria", "Las ocupaciones en las que trabajan sobre todo mujeres poseen peores condiciones laborales que en las que trabajan sobre todo hombres", "Las profesiones en las que trabajan sobre todo mujeres están peor remuneradas que las profesiones en las que trabajan sobre todo hombres" y "En nuestra sociedad las mujeres no sufren discriminación a la hora de incorporarse al mercado laboral".

Por el contrario, los chicos obtienen puntuaciones medias algo más elevadas que las chicas en los ítems "Contratar a las mujeres solo trae problemas a las empresas" y "Hay trabajos en los que los hombres deberían tener preferencia sobre las mujeres a la hora de los ascensos y de la promoción". Aunque se manifiestan en desacuerdo con estas afirmaciones lo hacen en menor medida que sus compañeras que manifiestan un mayor grado de desacuerdo.

La postura ante los estereotipos de género de los alumnos no parece tan clara, en comparación con las alumnas, al situar sus respuestas en torno al punto medio de la escala en las afirmaciones: "Hay trabajos que son más apropiados para las mujeres que para los hombres" y viceversa "Hay trabajos que son más apropiados para los hombres que para las mujeres", "Hay trabajos en los que se necesita fuerza y otras cualidades/habilidades que las mujeres no tienen" y "Hay algunos tipos de trabajos más apropiados para las mujeres y otros más apropiados para los hombres".

Tabla 3

Diferencias por sexo en los estereotipos de género

\begin{tabular}{|c|c|c|c|c|c|c|}
\hline \multirow{2}{*}{ Estereotipos de Género } & \multicolumn{2}{|c|}{ Mujeres } & \multicolumn{2}{|c|}{ Hombres } & \multirow{2}{*}{$\mathbf{t}$} & \multirow{2}{*}{$\mathrm{p}$} \\
\hline & $(\bar{X})$ & $(s)$ & $(\bar{X})$ & $(s)$ & & \\
\hline $\begin{array}{l}\text { Las mujeres deben tener las mismas } \\
\text { posibilidades de trabajo que los hombres. }\end{array}$ & 4.86 & 0.655 & 4.44 & 1.283 & 0.4361 & .000 \\
\hline $\begin{array}{l}\text { Contratar a las mujeres solo trae problemas a } \\
\text { las empresas. }\end{array}$ & 1.10 & 0.519 & 1.46 & 1.082 & -4.591 & .000 \\
\hline $\begin{array}{l}\text { Hay trabajos que son más apropiados para las } \\
\text { mujeres que para los hombres. }\end{array}$ & 2.26 & 1.238 & 2.83 & 1.444 & -4.514 & .000 \\
\hline $\begin{array}{l}\text { Hay trabajos que son más apropiados para } \\
\text { los hombres que para las mujeres. }\end{array}$ & 2.34 & 1.307 & 3.03 & 1.491 & -5.207 & .000 \\
\hline $\begin{array}{l}\text { Hay trabajos en los que los hombres deberían } \\
\text { tener preferencia sobre las mujeres a la hora } \\
\text { de los ascensos y de la promoción. }\end{array}$ & 1.28 & 0.802 & 1.67 & 1.141 & -4.280 & .000 \\
\hline $\begin{array}{l}\text { Las mujeres tienen más dificultades para } \\
\text { encontrar un trabajo que tradicionalmente se } \\
\text { identifica como un trabajo de hombres. }\end{array}$ & 4.20 & 0.906 & 3.49 & 1.478 & 6.235 & .000 \\
\hline
\end{tabular}


Los hombres tienen más dificultades para encontrar un trabajo que tradicionalmente se identifica como un trabajo de mujeres.

$\begin{array}{llllll}3.43 & 1.262 & 3.16 & 1.425 & 2.145 & .032\end{array}$

Las ocupaciones en las que trabajan sobre todo hombres tienen mayor reconocimiento social que las ocupaciones en las que la presencia femenina es mayoritaria.

Las ocupaciones en las que trabajan sobre todo mujeres poseen peores condiciones laborales que las ocupaciones en las que trabajan sobre todo hombres.

Las profesiones en las que trabajan sobre todo mujeres están peor remuneradas que las profesiones en las que trabajan sobre todo hombres

Hay trabajos en los que se necesita fuerza y otras cualidades/habilidades que las mujeres no tienen.

Hay algunos tipos de trabajos más apropiados para las mujeres y otros más apropiados para los hombres.

En nuestra sociedad las mujeres no sufren discriminación a la hora de incorporarse al mercado laboral.

$\begin{array}{llllll}3.19 & 1.341 & 2.67 & 1.331 & 4.139 & .000\end{array}$

$\begin{array}{llllll}2.99 & 1.361 & 2.45 & 1.316 & 4.227 & .000\end{array}$

$\begin{array}{llllll}3.56 & 1.226 & 2.74 & 1.365 & 6.673 & .000\end{array}$

$\begin{array}{llllll}2.76 & 1.321 & 3.20 & 1.523 & -3.293 & .001\end{array}$

$\begin{array}{llllll}2.87 & 1.388 & 3.32 & 1.477 & -3.358 & .001\end{array}$

$\begin{array}{llllll}3.30 & 1.288 & 2.56 & 1.422 & 5.757 & .000\end{array}$

\section{Diferencias en los estereotipos de género según ciclo formativo de grado medio o superior}

Analizamos también si existían diferencias en función de si el ciclo formativo es de grado medio o superior. Al respecto, los resultados derivados de la prueba t de Student muestran la existencia de diferencias estadísticamente significativas en los ítems de la escala "Hay trabajos que son más apropiados para los hombres que para las mujeres", "Hay trabajos en los que los hombres debería tener preferencia sobre las mujeres a la hora de los ascensos y de la promoción", "Los hombres tienen más dificultades para encontrar un trabajo que tradicionalmente se identifica como un trabajo de mujeres" y "Hay algunos tipos de trabajos más apropiados para las mujeres y otros más apropiados para los hombres".

Estas diferencias son, en todos los casos, a favor del alumnado que cursa ciclos formativos de grado medio, excepto en el ítem "Los hombres tienen más dificultades para encontrar un trabajo que tradicionalmente se identifica como un trabajo de mujeres", en el cual los estudiantes que cursan ciclos formativos de grado superior obtienen puntuaciones medias más altas, expresando un mayor grado de acuerdo con esta afirmación (ver tabla 4). 
Tabla 4

Diferencias en los estereotipos de género según ciclo formativo de grado medio o superior

\begin{tabular}{|c|c|c|c|c|c|c|}
\hline \multirow{2}{*}{ Estereotipos de Género } & \multicolumn{2}{|c|}{$\begin{array}{l}\text { Grado } \\
\text { Medio }\end{array}$} & \multicolumn{2}{|c|}{$\begin{array}{l}\text { Grado } \\
\text { Superior }\end{array}$} & \multirow{2}{*}{$\mathbf{t}$} & \multirow{2}{*}{$\mathbf{p}$} \\
\hline & $(\bar{X})$ & $(s)$ & $(\bar{X})$ & $(S)$ & & \\
\hline $\begin{array}{l}\text { Hay trabajos que son más apropiados para los hom- } \\
\text { bres que para las mujeres. }\end{array}$ & 2.88 & 1.454 & 2.48 & 1.397 & 2.981 & .030 \\
\hline $\begin{array}{l}\text { Hay trabajos en los que los hombres deberían tener } \\
\text { preferencia sobre las mujeres a la hora de los ascen- } \\
\text { sos y de la promoción. }\end{array}$ & 1.60 & 1.092 & 1.30 & 0.886 & 2.765 & .006 \\
\hline $\begin{array}{l}\text { Los hombres tienen más dificultades para encon- } \\
\text { trar un trabajo que tradicionalmente se identifica } \\
\text { como un trabajo de mujeres. }\end{array}$ & 3.18 & 1.418 & 3.42 & 1.276 & -1.923 & .055 \\
\hline $\begin{array}{l}\text { Hay algunos tipos de trabajos más apropiados para } \\
\text { las mujeres y otros más apropiados para los hombres. }\end{array}$ & 3.27 & 1.477 & 2.91 & 1.401 & 2.672 & .008 \\
\hline
\end{tabular}

\section{Diferencias por edad en los estereotipos de género}

Si tenemos en cuenta la edad de los sujetos de la muestra, el análisis de varianza realizado para cada uno de los ítems de nuestra escala, constata la no existencia de diferencias estadísticamente significativas en los distintos grupos de edad, de manera que no podemos confirmar con nuestros datos que los estereotipos de género se atenúen con la edad.

\section{Discusión y conclusiones}

Los resultados obtenidos en nuestro trabajo, al igual que en estudios similares, permiten afirmar que el alumnado muestra una mayor sensibilización hacia la igualdad (Alvariñas \& Novoa, 2016; García Pérez et al., 2010; Pastor \& Marrucci, 2015). En nuestro estudio esta realidad se vislumbra en el alumnado que cursa un ciclo formativo de grado medio o superior cuyas actitudes hacia el trabajo son cada vez más igualitarias, pues parecen haber interiorizado ciertas afirmaciones como, por ejemplo, que "Las mujeres deben tener las mismas posibilidades de trabajo que los hombres".

En nuestra opinión, estos hallazgos son congruentes con la situación actual de las mujeres en el mercado laboral y su presencia cada vez mayor en los diferentes sectores de actividad, siendo precisamente el ámbito laboral uno de los espacios donde se pueden observar mayores logros en materia de igualdad en los últimos años (Cebrián \& Moreno, 2008).

No obstante, cabe señalar la existencia de diferencias de género en los estereotipos. De este modo, las chicas manifiestan una mayor sensibilización hacia la igualdad que los chicos en aspectos relacionados con las condiciones laborales, el reconocimiento social y las dificultades de acceso al mercado laboral, algo similar a lo encontrado por García Pérez et al. (2010) y Pastor y Marrucci (2015) en sus respectivos estudios. 
Por el contrario, aunque se muestran a favor de la igualdad en el trabajo, los chicos parece que han interiorizado más que las chicas ciertos estereotipos de género cuando aluden a la idoneidad de una actividad laboral u otra dependiendo del sexo y cuando se refieren a las competencias que es necesario dominar para realizar ciertas actividades asociadas a un tipo de trabajo determinado.

Por otra parte, la edad no parece tener mayor repercusión en los estereotipos de género. Nuestros resultados muestran, a diferencia de otros estudios (Ruiz Palomino et al., 2010; Sánchez García et al., 2011), que los estereotipos no se modifican con la edad, quizás debido a que la mayoría de los sujetos de la muestra tienen edades comprendidas entre los 16 y 20 años.

Es evidente que algo está cambiando en la sociedad actual, pero, a pesar de que las relaciones intergrupales entre los géneros están evolucionando, todavía persisten ciertos estereotipos de género que se resisten al cambio.

Entre los cambios que se están produciendo cabe señalar el respaldo legal e institucional facilitador de medidas favorecedoras de la no discriminación por sexo en el mercado laboral, así como el incremento del número de mujeres que acceden a los estudios universitarios y su mayor presencia en puestos de liderazgo.

Al respecto, López-Zafra, García-Retamero, Diekman y Eagly (2008) señalan que el hecho de que mujeres y hombres asuman roles que no se ajustan a lo socialmente esperado para su sexo, modifica la percepción que se tiene de los estereotipos de género.

No obstante, sigue siendo necesario profundizar en el estudio de los mecanismos que subyacen a estos cambios para comprobar si son reales o son simplemente un mero reflejo de lo deseable socialmente. Es necesario, en palabras de Martínez Labrín y Bivort (2013, p.556) “trabajar con niñas y niños, incluir este trabajo en los currículos en la formación inicial de docentes, trabajar en los componentes de la estructura educacional y reforzar a quienes participan de la educación informal".

\section{Referencias}

Alvariñas, M., Fernández Villarino, M.A., \& López Villar, C. (2009). Actividad física y percepciones sobre deporte y género. Revista de Investigación en Educación, 6, 113-122. Recuperado de http://reined.webs.uvigo.es/ojs/index.php/reined/article/ viewFile $/ 58 / 52$

Alvariñas, M., \& Novoa, A. (2016). Pensamientos relacionados con la actividad física y el género en adolescentes de Galicia. Sportis. Revista Técnico-Científica del Deporte Escolar, Educación Física y Psicomotricidad, 2(1), 23-35. doi: 10.17979/sportis.2016.2.1.1439

Bem, S. L. (1981). Gender schema theory: A cognitive account of sex typing. Psychological Review, 88(4), 354-364. doi: 10.1037/0033-295X.88.4.354

Castillo-Mayén, M.R., \& Montes-Berges, B. (2014). Analysis of current gender stereotypes. Anales de psicología, 30(3), 1044-1060. doi: 10.6018/analesps.30.3.138981

Cebrián, I., \& Moreno, G. (2008). La situación de las mujeres en el mercado de trabajo español: desajustes y retos. Economía Industrial, 367, 121-137. Recuperado de http:// www.minetur.gob.es/Publicaciones/Publicacionesperiodicas/EconomiaIndustrial/ RevistaEconomiaIndustrial/367/121.pdf 
Colás, P., \& Villaciervos, P. (2007). La interiorización de los estereotipos de género en jóvenes y adolescentes. Revista de Investigación Educativa, 1(25), 35-58. Recuperado de http://revistas.um.es/rie/article/view/96421/92631

de Lemus, S., Castillo, M., Moya, M., Padilla, J.L., \& Ryan, E. (2008). Elaboración y validación del inventario de sexismo ambivalente para adolescentes. International Journal of Clinical and Health Psychology, 8(2), 537-562. Recuperado de http://www. redalyc.org/pdf/337/33712001013.pdf

Delpino, M., \& Eresta, M. (2013). Relaciones afectivas y sexualidad en la adolescencia. Madrid: Ministerio de Sanidad, Servicios Sociales e Igualdad.

Díaz-Aguado, M. J., Martínez Arias, R., \& Martínez Babarro, J. (2014). La evolución de la adolescencia española sobre la igualdad y la prevención de la Violencia de género. Madrid: Ministerio de Sanidad, Política Social e Igualdad. Centro de Publicaciones.

Elejabeitia, C., \& López Sáez, M. (2003). Trayectorias personales y profesionales de mujeres con estudios tradicionalmente masculinos. Madrid: Instituto de la Mujer y CIDE.

García Pérez, R., Rebollo, M.A., Buzón, O., González-Piñal, R., Barragán, R., \& Ruíz Pinto, E. (2010). Actitudes del alumnado hacia la igualdad de género. Revista de Investigación Educativa, 28(1), 217-232. Recuperado de http://revistas.um.es/rie/ article/view/98951

Heilman, M. (2015). Gender stereotypes: Impediments to women's career progress. En I. Welpe, P. Brosi, L. Ritzehöfer, \& T. Schwarzmüller (Eds.), Auswhal von Männern und Frauen als Führungskräfte. Perspektiven aus Wirtschaft, Wissenschaft, Medien und Politik (pp. 73-84). doi: 10-1007/978-3-658-09469-0_1

Igbo, J.N., Onu, V.C., \& Obiyo, N.O. (2015). Impact of gender stereotype on secondary school students 'self-concept and academic achievement. Sage Open, 5(1), 1-10. doi: $10.1177 / 2158244015573934$

Kurtz-Costes, B., Copping, K.E., Rowley, S.J., \& Kinlaw, C.R. (2014). Gender and age differences in awareness and endorsement of gender stereotypes about academic abilities. European Journal of Psychology of education, 29(4), 603-618. doi: 10.1007/ s10212-014-0216-7

López-Zafra, E., García-Retamero, R., Diekman, A., \& Eagly, A. H. (2008). Dinámica de estereotipos de género y poder: un estudio transcultural. Revista de Psicología Social, 23(2), 213-219. doi: 10.1174/021347408784135788

Markus, H., Crane, H., Bernstein, S., \& Siladi, M. (1982). Self-schemas and gender. Journal of Personality and Social Psychology, 42(1), 38-50. doi: 10.1037/0022-3514.43.6.1192

Martínez Labrín, S.L., \& Bivort, B.U. (2013). Los estereotipos en la compensación de las desigualdades de género en educación, desde la psicología feminista. Psicología y Sociedad, 25(3), 549-558. Recuperado de http://www.scielo.br/pdf/psoc/v25n3/09.pdf

Miller, D.I., Eagly, A.H., \& Linn, M.C. (2015). Women's representation in science predicts national gender-science stereotypes: Evidence from 66 nations. Journal of Educational Psychology, 107(3), 631-644. doi: 10.1037/edu0000005

Pastor, L., \& Marrucci, C. (2015). Valores y estereotipos de género presentes en adolescentes italianos y españoles. En Asociación Interuniversitaria de Investigación Pedagógica, AIDIPE (Ed.), Investigar con y para la sociedad (pp. 443-454). Madrid: AIDIPE.

Perry, D., \& Pauletti, R. (2011). Gender and adolescent development. Journal of Research on Adolescence, 21(1), 61-74. doi: 10.1111/j.1532-7795.2010.00715.x 
Porto, A.M., Cajide, J., Mosteiro, M. J., Castro, M. D., Sierra, S., \& Rodríguez Burgos, S. (2012). Estereotipos de género ante la Ciencia y la Tecnología del alumnado de Formación Profesional. Actas del IX Congreso Iberoamericano de Ciencia, Tecnología y Género, 1-13. Recuperado de http://www. oei.es/congresoctg/memoria/pdf/2PortoCastro.pdf .

Rodríguez San Julián, E., \& Megías, I. (2015). ¿Fuerte como papá? ¿Sensible como mamá?. Identidad de género en la adolescencia. Madrid: Centro Reina Sofía sobre Adolescencia y Juventud.

Ruiz Palomino, E., Ballester, R., Gil Llario, M.D., Giménez García, C., \& Salmerón, P. (2010). ¿Cambian los estereotipos de género a lo largo de la adolescencia?. International Journal of Developmental and Educational Psychology, 1(2), 321-329. Recuperado de http://hdl.handle.net/10234/31616

Sánchez García, M., Suárez Ortega, M., Manzano, N., Oliveros, L., Lozano, S., Fernández D'Andrea, B., \& Malik, B. (2011). Estereotipos de género y valores sobre el trabajo entre los estudiantes españoles. Revista de Educación, 355, 331-354. Recuperado de http://www.revistaeducacion.educacion.es/re355/re355_14.pdf

Vázquez-Cupeiro, S. (2015). Ciencia, estereotipos y género. Una revisión de los marcos explicativos. Convergencia: Revista de ciencias sociales, 22(68), 177-202. Recuperado de http://www.redalyc.org/articulo.oa?id=10536227007

Fecha de recepción: 22 de abril de 2016

Fecha de revisión: 22 de abril de 2016

Fecha de aceptación: 19 de mayo de 2016 
